\title{
UX Monocerotis as a W Serpentis binary
}

\author{
D. Sudar ${ }^{1}$, P. Harmanec ${ }^{2}$, H. Lehmann ${ }^{3}$, S. Yang ${ }^{4}$, H. Božić ${ }^{1}$, and D. Ruždjak ${ }^{1}$ \\ ${ }^{1}$ Hvar Observatory, Faculty of Geodesy, Kačićeva 26, University of Zagreb, 10000 Zagreb, Croatia \\ e-mail: dsudar@geof.hr \\ 2 Astronomical Institute of the Charles University, Faculty of Mathematics and Physics, V Holešovičkách 2, 18000 Praha 8 , \\ Czech Republic \\ 3 Thüringer Landessternwarte Tautenburg, 07778 Tautenburg, Germany \\ ${ }^{4}$ Dept. of Physics and Astronomy, University of Victoria, PO Box 3055, Victoria BC V8W 3P6, Canada
}

Received 3 May 2010 / Accepted 15 January 2011

\begin{abstract}
Using our new photometric and spectroscopic observations as well as all available published data, we present a new interpretation of the properties of the peculiar emission-line binary UX Mon. We conclude that this binary is in a rare phase of fast mass transfer between the binary components prior to the mass ratio reversal. We firmly establish that the orbital period is secularly decreasing at a rate of $\dot{P}=-0.260$ s per year. From several lines of reasoning, we show that the mass ratio of the component losing mass to the mass-gaining component $q$ must be larger than 1 and find our most probable value to be $q=1.15 \pm 0.1$. The BINSYN suite of programs and the steepest descent method were used to perform the final modeling. We modeled the star as a W Ser star with a thick disk around its primary. Although the remaining uncertainties in some of the basic physical elements describing the system in our model are not negligible, the model is in fair agreement with available observations. Only the nature of the light variations outside the primary eclipse remains unexplained.
\end{abstract}

Key words. stars: emission-line, Be - binaries: close - stars: individual: UX Monocerotis - binaries: eclipsing stars: fundamental parameters

\section{Introduction}

UX Monocerotis (HD 65607, SAO 135333) is an eclipsing binary with an orbital period of 5 d.90. Its binary nature was first reported by Woods \& Shapley (1928), who also noted that its brightness at maximum light is not constant. Gaposchkin (1947) studied 32 spectrograms from the McDonald Observatory and 1389 photographic plates from the Harvard Observatory. He identified the star eclipsed at the deeper minimum (hereafter, the primary) as an $\mathrm{A} 7 \mathrm{p}$ object and the other component (hereafter, the secondary) as a G2p star. He arrived at surprisingly low masses of $M_{1}=0.73 M_{\odot}$ and $M_{2}=0.74 M_{\odot}$ for the primary and the secondary, respectively. Struve (1947) analyzed 152 photographic spectra secured in 1947 . He obtained radialvelocity (RV hereafter) semi-amplitudes of $K_{1}=140 \mathrm{~km} \mathrm{~s}^{-1}$ and $K_{2}=60 \mathrm{~km} \mathrm{~s}^{-1}$ for the primary and secondary, respectively. This implies a more massive secondary, $M_{2} \sin ^{3} i=3.4 M_{\odot}$, than the primary, $M_{1} \sin ^{3} i=1.5 M_{\odot}$, which would mean that UX Mon is in the rare evolutionary stage prior to the mass reversal (cf. e.g. Crawford 1955; Morton 1960). Struve also discussed the origin of the Balmer emission, which he identified as circumstellar matter located between the two stars.

Hiltner et al. (1950) published 486 yellow photoelectric observations with an effective wavelength of $5300 \AA$. They confirmed the light variations outside the primary eclipse. They also noted that the durations of both eclipses are the same. Wood (1957) obtained light curves of the system in the yellow and blue filters. He observed short (hours) and long (six or more days) non-orbital light changes. Lynds (1956, 1957a,b) secured and analyzed $333 U B V$ observations. He derived the ratio of radii, $k=0.522$, the radius of the secondary relative to the orbital separation, $r_{2}=0.358$, and the inclination, $i=83^{\circ} .6$.

Scaltriti $(1973,1976)$ concluded that the primary is a $\delta$ Scuti variable and that there had been an abrupt change of the orbital period in the past.

Umana et al. (1991) found that the UX Mon system is a microwave source and concluded that the microwave radiation originates from the gyrosynchrotron radiation of electrons in a magnetic field.

Olson \& Etzel (1995) carried out a detailed photometric and spectroscopic study of UX Mon based on their new ubvyI observations and a series of electronic spectra. They derived the most accurate RV semi-amplitude of the secondary yet obtained, $K_{2}=108.3 \pm 1.9 \mathrm{~km} \mathrm{~s}^{-1}$, but were unable to detect any measurable spectral lines of the primary. In contrast to Struve (1947), they inferred a mass ratio secondary/primary of $q=0.8$, based on light curves analysis. Their model also led to the surface temperatures $T_{1} \approx 8000 \mathrm{~K}$ and $T_{2} \approx 5500 \mathrm{~K}$ for the primary and secondary, respectively. They concluded that the gas stream from the secondary hits the primary directly, without forming an accretion disk around it.

Analyzing published photometry and spectroscopy, Ondřich \& Harmanec (2003) concluded that the orbital period of UX Mon has been secularly decreasing and provided new quadratic ephemeris. They also discussed the emission lines of highly ionized atoms in the HST/GHRS spectra indicating that UX Mon is a W Ser star. W Ser stars are strongly interacting binaries characterized by the presence of numerous emission lines in their UV spectra, which are believed to originate 
Table 1. Journal of photometric observations of UX Mon.

\begin{tabular}{lccc}
\hline \hline Sources & $\begin{array}{c}\text { Time interval } \\
\text { (HJD-2 400 000) }\end{array}$ & $\begin{array}{c}\text { No. of. } \\
\text { obs }\end{array}$ & Passbands \\
\hline$(1)$ & $18600.0-18605.7$ & 38 & vis \\
$(2)$ & $33246.9-33381.6$ & 486 & $\lambda 5300$ \\
$(3)$ & $33300.8-33399.7$ & 401 & $\lambda 5200, \lambda 4250$ \\
$(4)$ & $35084.9-35153.9$ & 999 & $U B V$ \\
$(5)$ & $41626.7-41764.3$ & 875 & $\lambda 5150, V$ \\
$(6)$ & $47181.7-48656.9$ & 5056 & $u v b y I$ \\
$(7)$ & $47964.6-48938.2$ & 76 & $V$ \\
$(8)$ & $51868.8-53896.5$ & 268 & $V$ \\
$(9)$ & $52655.4-54219.3$ & 402 & $U B V$ \\
$(10)$ & $52655.4-54219.3$ & 137 & vis \\
$(11)$ & $51448.0-54228.6$ & 1453 & $B V$ \\
\hline
\end{tabular}

References. (1) Woods \& Shapley (1928); (2) Hiltner et al. (1950); (3) Wood (1957); (4) Lynds (1957a); (5) Scaltriti (1973); (6) Olson \& Etzel (1995); (7) Perryman \& ESA (1997); (8) Pojmański (1997); (9) Hvar observations (this paper); (10) Meyer (priv. comm.); (11) Olson et al. (2009).

in an extended gaseous envelope around their mass-gaining components (cf. e.g. Plavec \& Koch 1978; Plavec 1980, 1992). However, the mass transfer rate $\left(10^{-8}-10^{-9} M_{\odot} \mathrm{y}^{-1}\right)$, which Ondřich \& Harmanec (2003) derived for UX Mon from the quadratic ephemeris, appears to be too low compared to the typical values of $10^{-6} M_{\odot} \mathrm{y}^{-1}$ measured for W Ser binaries (Plavec 1980, 1992). Independently, the secular period decrease was also noted by Meyer (2006).

Olson et al. (2009) published a new study of UX Mon based on systematic photometry secured over a period of eight years. They found almost the same system parameters as in their previous paper (Olson \& Etzel 1995). Focusing mainly on light variations outside the primary eclipse, they concluded that these are probably caused by the variable mass transfer.

\section{Observations}

\subsection{Photometry}

Altogether, 22 different datasets of photometric observations of UX Mon from 11 different sources were used. Table 1 lists basic observational data. More information about the datasets used in this paper is given below.

(1) Photographic photometry, consisting of 38 phase-averaged normal points published by Woods \& Shapley (1928) only in orbital phases. The accurate dates of observations are unknown limiting their usefulness in our study.

(2) These observations were obtained at McDonald Observatory from November 26, 1949 to April 10, 1950.

(3) Wood (1957) obtained 401 photoelectric observations, divided into two (green and blue) datasets.

(4) There are 333 individual observations in each of the $U B V$ passbands in this dataset, obtained during twenty nights between December 7, 1954, and February 15, 1955. The instrumental color system is essentially the same as the standard $U B V$ system, except for the zero point.

(5) Two datasets of green and yellow photoelectric observations contain a total of 875 individual observations.

(6) These five sets of uvbyI(Kron) observations consist of 5056 observations in total. They were secured between January 21, 1988, and February 4, 1992 and analyzed by Olson \& Etzel (1995). Upon our request, Dr. Olson kindly allowed us to have copies of these observations at our disposal. These data were subsequently published by Olson et al. (2009).

(7) The Hipparcos $H_{\mathrm{p}}$ photometry of UX Monocerotis was extracted from the data archive published by Perryman \& ESA (1997) and transformed to the standard Johnson $V$ magnitude using the transformation formulae derived by Harmanec (1998) and Harmanec \& Božić (2001), which are based on numerous all-sky $U B V$ observations of many stars observed at Hvar.

(8) All-Sky Automated Survey (ASAS hereafter) is an observational program for monitoring stars of the southern hemisphere brighter than $\approx 14$ th magnitude (Pojmański 1997, 2001). Altogether, 268 Johnson $V$ observations of UX Mon were secured between November 20, 2000 and June 10, 2006.

(9) Altogether, 402 new photoelectric $U B V$ observations were secured during two seasons with the 0.65-m Cassegrain reflector of the Hvar Observatory. The transformation from the instrumental to the standard Johnson system was carried out by the program HEC22 (Harmanec et al. 1994), which uses non-linear transformation formulae. The most developed version rel.16.2 was used, which allows modeling of variable extinction during the observing nights.

(10) This dataset consists of visual estimates by Meyer (2006), Meyer (priv. comm.)

(11) Olson et al. (2009) obtained 1453 individual measurements in Johnson's $B$ and $V$ filters with the $0.4 \mathrm{~m}$ APT at Fairborn Observatory in southern Arizona, USA.

\subsection{Spectroscopy}

In total, 39 new electronic spectra obtained at three observatories are included in this paper.

(1) Six spectra were obtained at the coudé focus of the Ondřejov Observatory 2.0-m reflector with a linear dispersion of $17 \AA \mathrm{mm}^{-1}$ (two-pixel resolution 12600), which cover a spectral range 6300-6700 A. Subtraction of bias, flatfielding, creation of $1 \mathrm{D}$ spectra, and wavelength calibration has been routinely carried out shortly after the observations by Dr. M. Šlechta. Rectification, heliocentric correction of time and radial velocity, and the removal of cosmic spikes was carried out by DS with the help of the program SPEFO (Horn et al. 1996; Škoda 1996) in its form developed by the late Mr. J. Krpata.

(2) Thirteen spectra were obtained in the coudé focus of the 1.22-m reflector of the Dominion Astrophysical Observatory (DAO hereafter). Their linear dispersion is $10 \AA \mathrm{mm}^{-1}$ (twopixel resolution 21700), while the range of wavelengths is 6100-6700 ̊. The initial reduction of spectra (bias removal, flatfielding, and creation of 1D spectra) was carried out by SY using IRAF. The wavelength calibration and all subsequent reductions were carried out by DS in SPEFO.

(3) Twenty echelle spectra were obtained at the coudé focus of the $2.0-\mathrm{m}$ reflector of TLS Tautenburg. These spectra have the best linear dispersion of all three sets: $3.2 \AA \mathrm{mm}^{-1}$ (two-pixel resolution 63000). They cover the spectral range 4700-7085 $\AA$. All reductions, including the rectification and heliocentric corrections were carried out by HL using standard MIDAS packages and a special routine to determine the nightly instrumental RV zero points from a large number of telluric $\mathrm{O}_{2}$ lines in the spectra. 
Table 2. Times of the primary minima and values of the instantaneous orbital period as derived by FOTEL assuming locally linear ephemeris.

\begin{tabular}{lccr}
\hline \hline Source & $\begin{array}{c}\text { Epoch } \\
\text { (HJD-2 400 000) }\end{array}$ & $\begin{array}{c}\text { Period } \\
\text { (days) }\end{array}$ & $\begin{array}{r}\mathrm{O}-\mathrm{C}_{2} \\
\text { (days) }\end{array}$ \\
\hline$(2)$ & $33246.1720(092)$ & $5.905882(563)$ & -0.0053 \\
$(3)$ & $33299.3269(063)$ & $5.904718(520)$ & +0.0082 \\
$(4)$ & $35088.3882(047)$ & $5.904210(689)$ & -0.0208 \\
$(5)$ & $41630.6478(157)$ & $5.904829(920)$ & -0.0074 \\
$(6)$ & $47180.8866(007)$ & $5.904494(004)$ & -0.0028 \\
$(8)$ & $51869.0342(078)$ & $5.904404(038)$ & +0.0016 \\
$(9)$ & $53746.6472(038)$ & $5.904432(067)$ & +0.0042 \\
$(11)$ & $52772.4039(019)$ & $5.904378(013)$ & -0.0008 \\
\hline
\end{tabular}

Notes. The column $\mathrm{O}-\mathrm{C}_{2}$ gives the differences with respect to quadratic ephemeris (Eq. (4)).

\section{Analysis}

\subsection{Secular changes of the orbital period}

Ondřich \& Harmanec (2003) proposed that there had been a secular decrease of the orbital period. We decided to re-investigate their finding, using more photometric observations than they had at their disposal.

We first calculated the local linear ephemeris of the sources listed in Table 1 with a sufficient number of measurements by using the latest publicly available version of the program FOTEL (Hadrava 2004a). We allowed for the convergence of the epoch of the primary minimum and the local period. The results are summarized in Table 2. We also added a third column, $\mathrm{O}-\mathrm{C}_{2}$, listing differences between the local epochs and those calculated from the quadratic ephemeris (see below).

In FOTEL, there is also an option that allows us to derive a period change, $\dot{P}$, that exactly corresponds to a quadratic ephemeris, as one of the unknowns of the solution. As discussed by Harmanec \& Scholz (1993), a quadratic ephemeris given by

$T=T_{0}+P_{0} E+a E^{2}$,

holds for the times of minima, where $P_{0}$ denotes the value of the period at the time of reference minimum $T_{0}$. Treating the epoch $E$ as a real number characterizing the cycle and phase, and denoting $T$ as the time corresponding to $E$ (i.e. to the given orbital phase at that cycle), one can write the instantaneous orbital period, $P$, as

$P=\frac{\mathrm{d} T}{\mathrm{~d} E}=P_{0}+2 a E$

The instantaneous change of the period, $\dot{P}$, is then

$\dot{P}=2 a \frac{\mathrm{d} E}{\mathrm{~d} T}=\frac{2 a}{P}$.

This shows that the quadratic ephemeris leads to a linear change of the period with epoch but not with time. For instance, for negative values of $a$, the rate of the period change increases with time.

We therefore used the corresponding option in FOTEL to derive the quadratic ephemeris given in Table 3 by combining all datasets from Table 2.

The explicit expression for the quadratic ephemeris is

$T_{\text {min.I }}=$ HJD $2452666.1319+5.9044365 E-2.43 \times 10^{-8} E^{2}$,

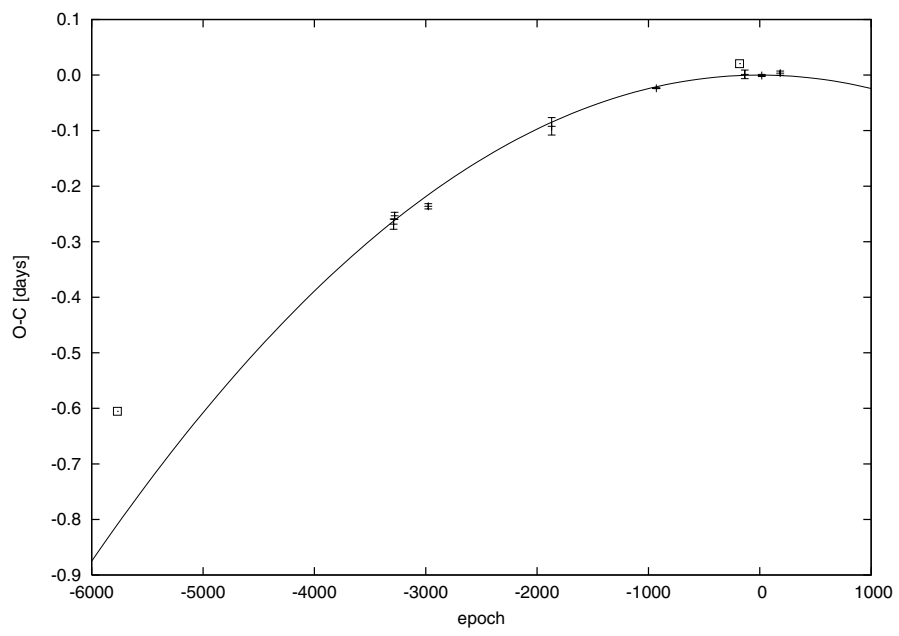

Fig. 1. O-C in days with respect to the linear ephemeris. Crosses with error bars denote the times of minima from Table 2 . The empty squares denote epochs of minima of sources (1) and (10). The times of individual observations for source (1) are unknown, and measurements from source (10) are of insufficient accuracy. Quadratic ephemeris is shown with a solid curve.

Table 3. The quadratic ephemeris, based on all photometric datasets of Table 2, derived with FOTEL.

\begin{tabular}{lc}
\hline \hline Quantity & Value \\
\hline$T_{\text {prim.min. }}$ [HJD] & $2452666.1319 \pm 0.0012$ \\
$P_{0}$ [days] & $5.9044365 \pm 0.0000020$ \\
$\dot{P}_{T=T_{0}}$ [days per day] & $(-8.23 \pm 0.18) \times 10^{-9}$ \\
$a$ [days] & $(-2.43 \pm 0.05) \times 10^{-8}$ \\
\hline
\end{tabular}

where the coefficient of the quadratic term is calculated as $P_{0} \dot{P}_{T=T_{0}} / 2$. The change of the orbital period corresponds to $\dot{P}_{T=T_{0}}=-0.260$ s per year.

The local epochs of minima from Table 2 were compared to the global linear ephemeris and the corresponding $\mathrm{O}-\mathrm{C}$ deviations are shown in Fig. 1. They clearly show the parabolic change indicating a secular decrease of the orbital period, in accordance with the findings of Ondřich \& Harmanec (2003).

Note that the epoch of the early photographic observations by Woods \& Shapley (1928) does not fit well with the extrapolated prediction of the quadratic ephemeris (Eq. (4)), but it also confirms that the period has been decreasing. Since the dates of these early observations are not known, we could not include this dataset into our FOTEL solution to obtain yet more accurate values for the quadratic ephemeris and the rate of the period change.

In Fig. 2 we fold all photometry near the primary minima from the datasets of Table 2 with the quadratic ephemeris (Eq. (4)), obtaining a very coherent fit. Although the depths of the minima differ, of course, for each passband, the phase of the primary mid-eclipse is the same for all of them.

Note that Ondřich \& Harmanec (2003) derived a smaller value of the period decrease, $\dot{P}=(-2.49 \pm 0.18) \times 10^{-9}$ days per day. For a fully conservative mass transfer, they estimated $\dot{M}=1.4 \times 10^{-9} M_{\odot}$ per year. We note, however, that their value refers to a mass transfer rate per one day, and that the correct mass transfer rate per one year should be $0.52 \mu M_{\odot} \mathrm{y}^{-1}$, i.e. typical of strongly interacting binaries (Plavec 1980). Our new result 


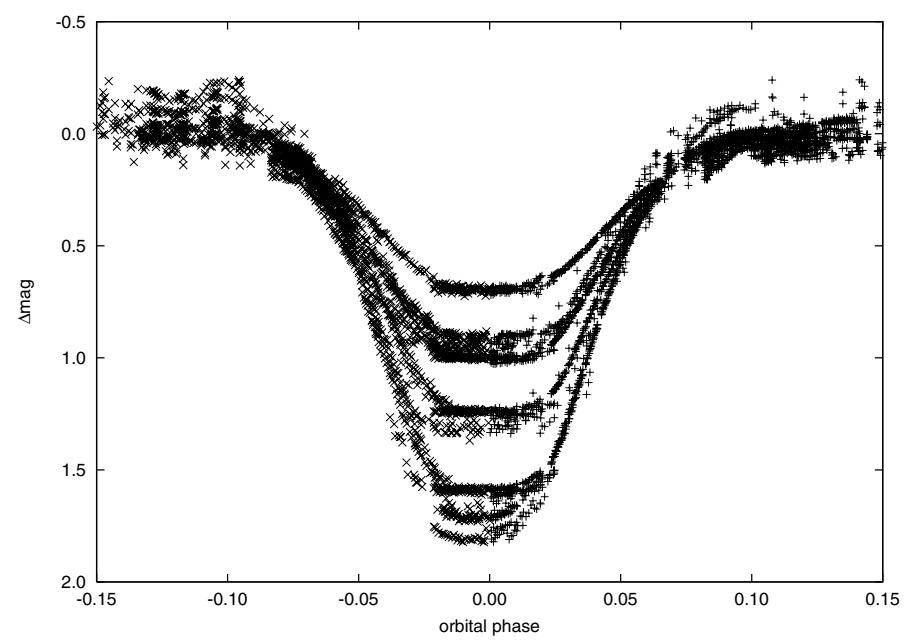

Fig. 2. A phase plot of all photometric observations around the phase of primary minimum using quadratic ephemeris (Eq. (4)).

implies a mass transfer rate that is even higher than the value by Ondřich \& Harmanec (2003, see below).

\subsection{Spectroscopic mass ratio}

The mass ratio of the binary systems with a Roche-lobe filling component can be estimated from the (plausible) assumption that the rotation of this star is synchronized with the orbital revolution (Andersen et al. 1989; Harmanec 1990). The essence of the method lies in the relative dimensions of the Roche lobe depending solely on the binary mass ratio $q$, while the absolute radius of the spin-orbit synchronized Roche-lobe filling star is given by the equatorial rotational velocity, the inclination of the rotational axis (assumed to be the same as the orbital inclination) and by the rotational period, which is identical to the binary orbital period. The third Kepler law is used to derive the binary separation.

To apply a similar procedure to UX Mon, we measured the rotational velocity of the secondary, $v_{2} \sin i$, using a mean spectrum constructed from three spectra closest to the phase of the primary mid-eclipse (phase $\phi=0$ ) and compared it with synthetic spectra. For that, a grid of model atmospheres was constructed using the LLmodels2 program (Shulyak et al. 2004), which allows us to calculate 1D plane-parallel, LTE model atmospheres for stars with effective temperatures of $5000 \mathrm{~K}$ and higher. Line absorption is taken into account directly for each line taken from a line list selected by the user. For the spectrum synthesis, we used the program SynthV (Tsymbal 1996).

To check whether the assumption of spin-orbit synchronism is in agreement with the value of $q=0.8$ obtained from photometry alone (Olson \& Etzel 1995; Olson et al. 2009), we adopted the values derived by the same authors: the temperature of the secondary $T_{2}=5500 \mathrm{~K}$ and its surface gravity acceleration $\log g=2.9$. Free parameters of the fit were microturbulent velocity, $\xi_{\mathrm{t}}$, and $v_{2} \sin i$. We also allowed for the adjustment of the local continuum of the observed spectrum to that of the synthetic one, compensating for possible large-scale misalignments due to an imperfect continuum placement during the spectrum reduction. To avoid the broad Balmer and stronger telluric lines, the analysis was restricted to the 4897 to $5777 \AA$ wavelength range. The best fit was obtained for $\xi_{\mathrm{t}}=(0.68+0.23) \mathrm{km} \mathrm{s}^{-1}$ and $v_{2} \sin i=90.7 \pm 3.0 \mathrm{~km} \mathrm{~s}^{-1}$. The $\chi^{2}$ values as a function of $v_{2} \sin i$ for various values of $\xi_{\mathrm{t}}$ are shown in Fig. 3 .

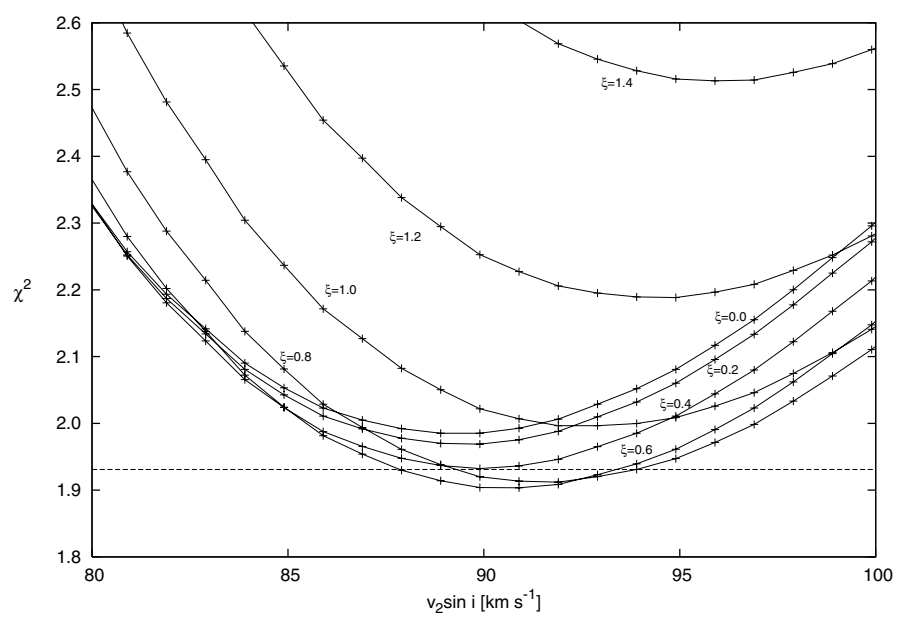

Fig. 3. $\chi^{2}$ value as a function of $v_{2} \sin i$ for various values of $\xi_{\mathrm{t}}$. A $95 \%$ confidence level is shown with a dashed line.

We used the value of $v_{2} \sin i=90.7 \pm 3.0 \mathrm{~km} \mathrm{~s}^{-1}$ and the orbital period $P=5.9044365$ days for the reference epoch derived in the previous section (first term in Eq. (4)). In addition, we used the inclination $i=89.5^{\circ}$ and the RV semi-amplitude of the orbital velocity of the secondary $K_{2}=108.3 \mathrm{~km} \mathrm{~s}^{-1}$ derived by Olson \& Etzel (1995). Following the numerical procedure presented in the appendix of Harmanec (1990) (see also the beginning of this section), we arrived at $q=1.3$. This estimate of $q$ differs substantially from the value of $q=0.8$ derived by Olson \& Etzel (1995). Note that we estimate the mass ratio again with the same method but using new values of all relevant parameters derived in this paper at the beginning of Sect. 4.

To find a more accurate value of the mass ratio, $q$, we used all of the spectra at our disposal (cf. Sect. 2.2). The RVs of individual unblended spectral lines were measured with SPEFO, which displays direct and reverse traces of the line profiles superimposed on the computer screen and the user can slide them to achieve their exact overlapping for the studied detail of the profile. The orbital phases were calculated with the quadratic ephemeris (Eq. (4)). Many suitable strong lines of the secondary could be identified. The RV curve of the secondary was calculated by performing a non-linear least squares fit assuming a circular orbit

$R V_{2}=(104.3 \pm 1.1) \sin (2 \pi \phi) \mathrm{km} \mathrm{s}^{-1}-0.2 \pm 0.8 \mathrm{~km} \mathrm{~s}^{-1}$

The resulting theoretical RV curve is plotted with the individual RV measurements in Fig. 4. A systematic deviation of the measured RVs from the calculated ones is visible before the secondary eclipse $(\phi=0.5)$. We attribute this difference to the Rossiter-McLaughlin effect. We return to this question in later sections.

To find spectral lines originating in the atmosphere of the primary proved to be a much more difficult task. A comparison of the spectra obtained near phases $\phi_{1}=0$ and $\phi_{2}=0.5 \mathrm{re}$ vealed that the most promising candidates are the following iron lines: Fe II $4923.921 \AA$, Fe II $5018.434 \AA$, and Fe II $5169.030 \AA$. However, the direct RV measurements with SPEFO, even for spectra from which the secondary lines with proper velocity shift were subtracted, resulted in a very uncertain RV curve displaying a large scatter. We, therefore, attempted the spectral disentangling using the latest publicly available version of the program KOREL (Hadrava 2004b). We concentrated on finding the best values of two parameters: the RV semi-amplitude of the 


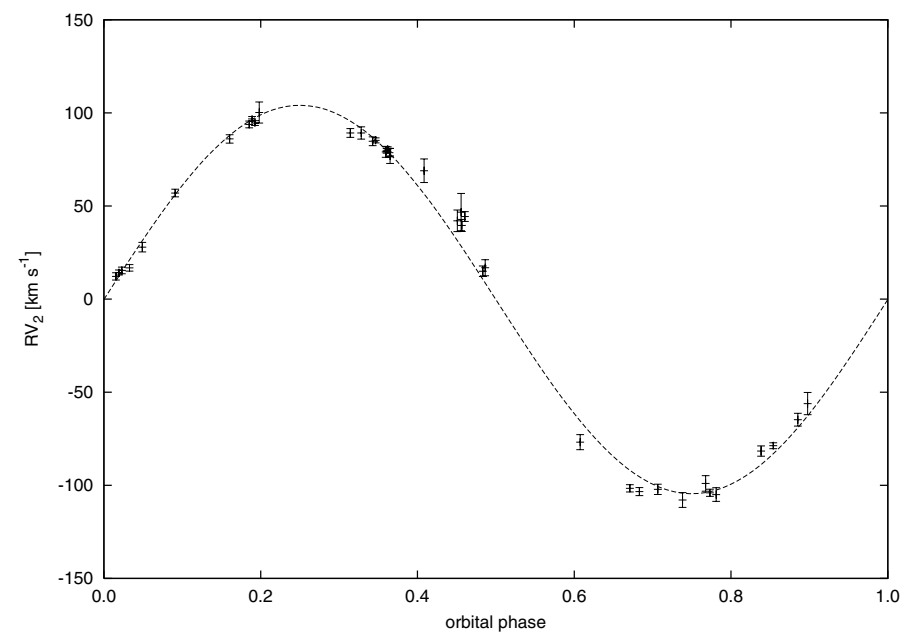

Fig. 4. The RV curve of the secondary given by Eq. (5) is shown by a dashed sinusoidal curve. The individual mean RVs of the secondary, based on SPEFO measurements, with their error bars are also shown.

secondary, $K_{2}$, and the mass ratio, $q$. We derived many solutions mapping the plausible range of both parameters. Since the value of $K_{2}$ is fairly well constrained by the solution given in Eq. (5) and by an independent result by Olson \& Etzel (1995) $\left(K_{2}=108.3 \mathrm{~km} \mathrm{~s}^{-1}\right)$, we only inspected the $K_{2}$ in the range from 100 to $112 \mathrm{~km} \mathrm{~s}^{-1}$. On the other hand, we treated the mass ratio as unconstrained over a wide range of values between 0.1 and 10 . We allowed convergence only for the time of the primary epoch and kept all other parameters fixed.

In Fig. 5, we plotted the sum of squares of the residuals, $s s r$, as a function of the two parameters $K_{2}$ and $q$ as a map plot. Although Fig. 5 represents only a crude mapping of the parameter space, it is quite clear (see Fig. 5a)) that the best-fit solution lies in the region $0.5<1 / q<1.0$.

To obtain more accurate values of the parameters $K_{2}$ and $q$, we selected 20 initial parameter sets with the lowest value of $s s r$ and allowed for the convergence of $K_{2}$ and $q$ while keeping everything else fixed. We obtained $K_{2}=104.6 \mathrm{~km} \mathrm{~s}^{-1}$ and $1 / q=0.87$ as the values of the lowest $s s r$. By estimating the error in $1 / q$ to be 0.05 and taking the error in $K_{2}$ from Eq. (5), we end up with

$$
\begin{aligned}
K_{2} & =104.6 \pm 1.1 \mathrm{~km} \mathrm{~s}^{-1} \\
q & =1.15 \pm 0.05 \\
K_{1} & =120.6 \pm 5.4 \mathrm{~km} \mathrm{~s}^{-1} \\
M_{1} \sin ^{3} i & =3.24 \pm 0.38 M_{\odot}, \\
M_{2} \sin ^{3} i & =3.74 \pm 0.28 M_{\odot}, \\
a \sin i & =26.29 \pm 0.64 R_{\odot} .
\end{aligned}
$$

Figure 6 shows the disentangled spectra of both components. The spectrum of the primary is shifted upwards by 0.2 for clarity. The three strong Fe II lines are clearly visible in the spectrum of the primary.

In the approximation of fully conservative mass transfer, a period decrease in time leads to the mass ratio $q>1$. Ondřich \& Harmanec (2003) carried a similar analysis and came to the same conclusion, challenging the value of $q=0.8$ published by Olson \& Etzel (1995). Our new value of $q=1.15$ agrees with their analysis.

Using the well-known formula for fully conservative mass transfer (cf. e.g. Harmanec \& Scholz 1993) and our estimates of
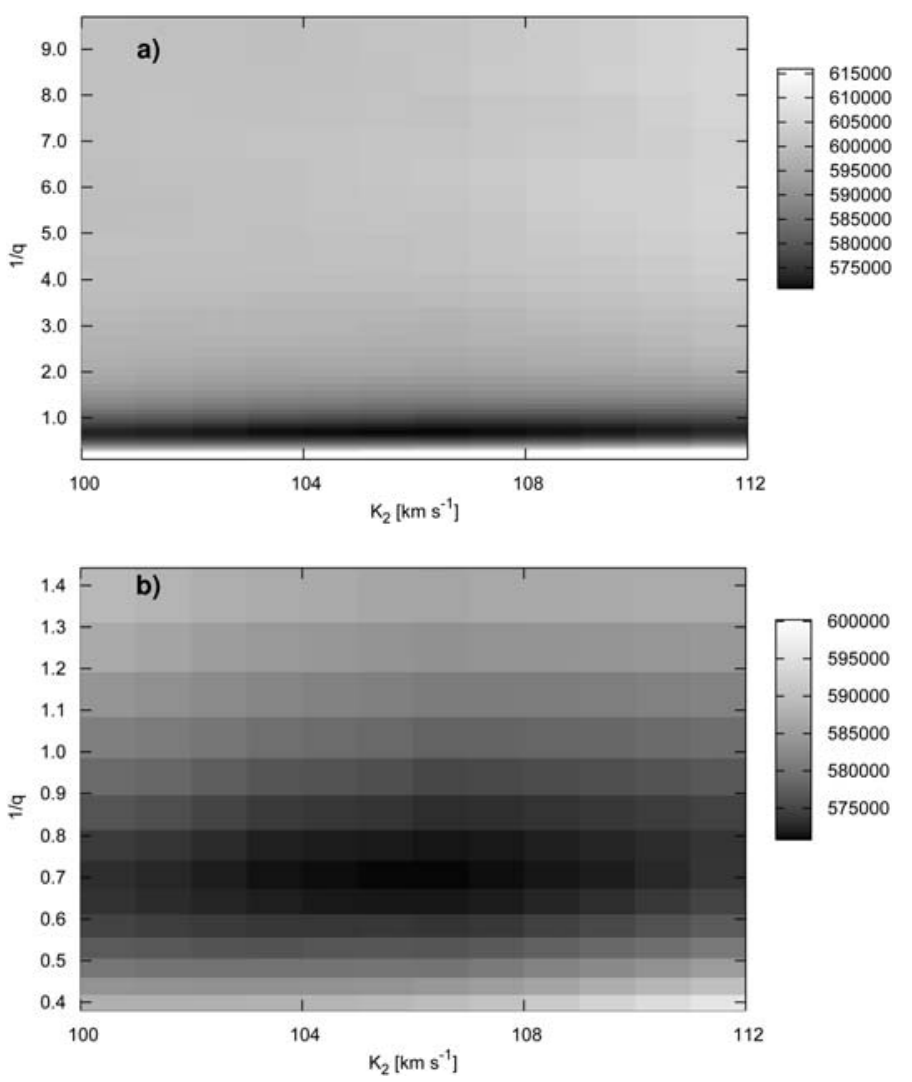

Fig. 5. a) Sum of squares of residuals, $s s r$, calculated with KOREL as a function of converged values of radial velocity semi-amplitude of the secondary, $K_{2}$, and inverse mass ratio $1 / q$ shown as a map plot. b) The same as in a) but with $1 / q$ axis scaled for better clarity.

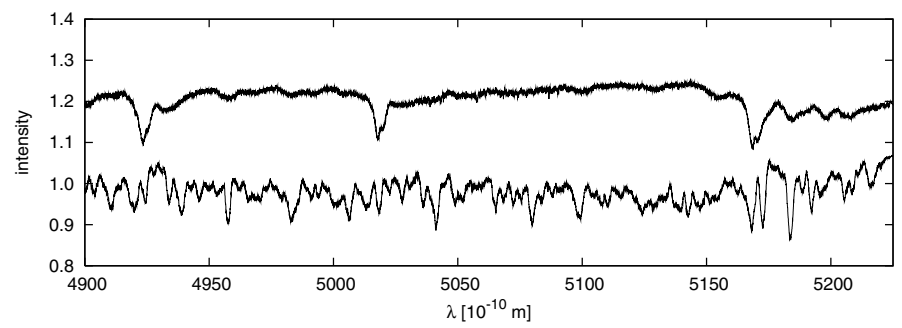

Fig. 6. The disentangled spectra of the two components.

the masses from the RV measurements, we obtain a mass transfer rate of $\dot{M}=(-3.6 \pm 1.3) \mu M_{\odot} \mathrm{y}^{-1}$. This value for the mass transfer rate is typical of W Ser stars (Plavec 1980, 1992).

We also note that the region around $\mathrm{H} \alpha$ exhibits a strong emission at all phases (Fig. 7). All 39 available spectra are shown. One can see that changes in the $\mathrm{H} \alpha$ profile are linked to orbital motion of the primary and secondary, shown in Fig. 7 by solid and dotted sinusoids, respectively.

\subsection{Light curve modeling}

Considering that mass transfer is present in the system and that Ondřich \& Harmanec (2003) found the emission lines of Si IV around $1400 \AA$ and of C IV around $1550 \AA$ in the UX Mon spectra, we felt encouraged to model the system as a W Ser star. This required us to model an optically and geometrically thick disk surrounding the primary star. 


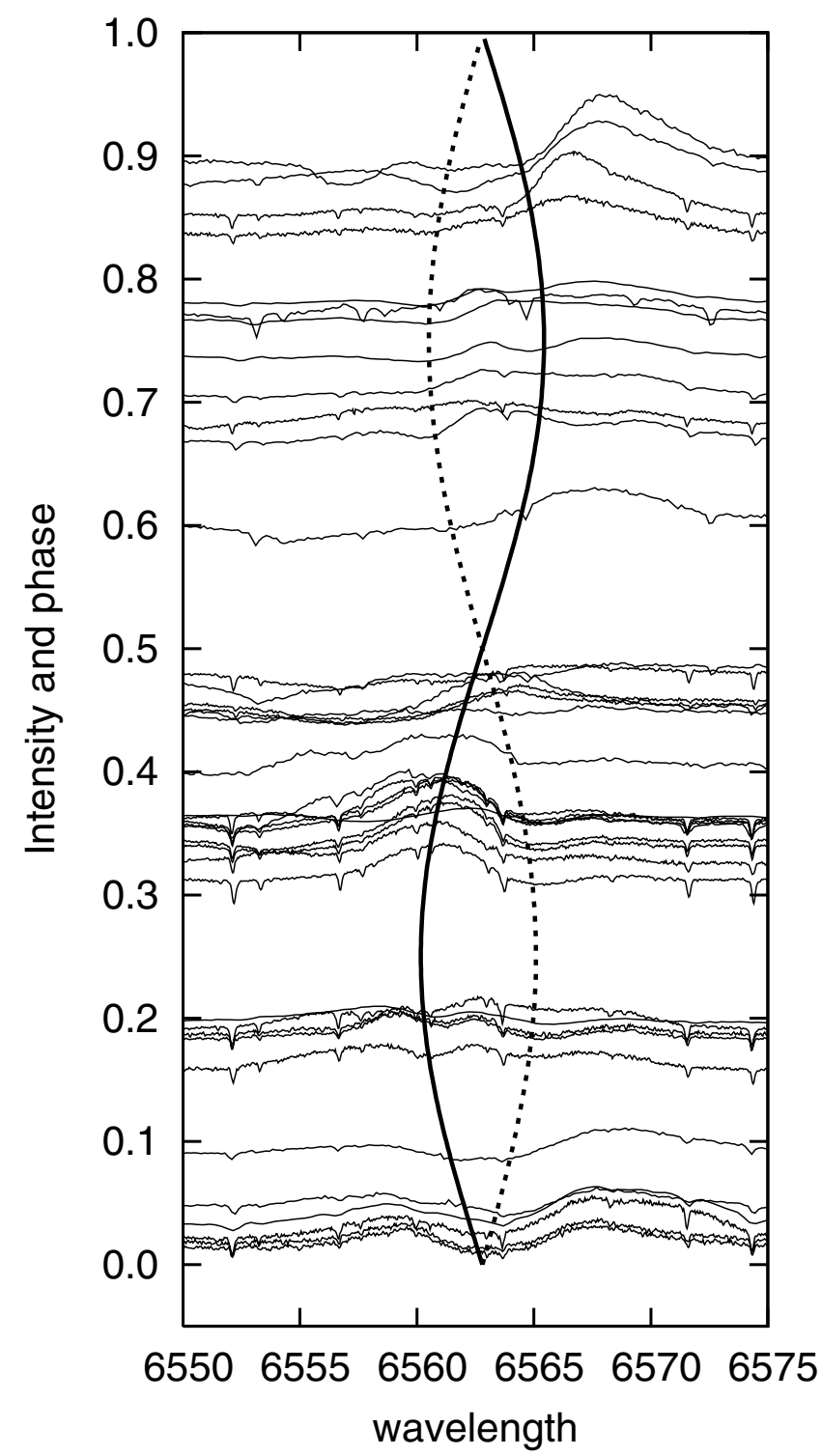

Fig. 7. A plot of all $39 \mathrm{H} \alpha$ profiles available to us, arranged according to their orbital phases (via appropriate shift of their continua along the ordinate labeled with the orbital phases). The solid and dotted sinusoids represent the orbital motion of the primary and secondary, respectively.

We used the BINSYN suite of programs (Linnell 1984; Linnell \& Hubeny 1996; Linnell 2000) with a parameter optimization procedure based on the steepest descent method developed by DS. The optimization procedure controlled the flow of BINSYN software, automating the process.

Using the results from the previous section, we kept $q=1.15$ and $M_{1} \sin ^{3} i=3.24 M_{\odot}$ constant. As can be seen from the light curve, the eclipse is total or close to totality. Therefore, we can estimate the mass of the primary to be in the range of $3.24 M_{\odot}$ to $3.4 M_{\odot}$. Assuming that the primary is a main-sequence star, we estimate its radius $R_{1} \approx 3 R_{\odot}$ and temperature $T_{1} \approx 13000 \mathrm{~K}$ (Harmanec 1988). Note that this assumption probably underestimates the actual radius because such large stars in close binaries are probably inflated with respect to their main-sequence counterparts.

Although the effect of the mass transfer can easily be seen from the secularly changing period, its effect on the physical parameters of the system (e.g. masses and separation between
Table 4. Initial and final values of the system parameters obtained with BINSYN. Errors of first four listed parameters were taken from Table 3 and Eq. (6).

\begin{tabular}{lccc}
\hline \hline Parameter & Initial value & Converged value & Error \\
\hline$P_{0}[$ days $]$ & 5.9044365 & - & 0.0000020 \\
$M_{1} \sin ^{3} i\left[M_{\odot}\right]$ & 3.24 & - & 0.38 \\
$a \sin i\left[R_{\odot}\right]$ & 26.29 & - & 0.64 \\
$q$ & 1.15 & - & 0.05 (est.) \\
$e$ & 0.0 & - & - \\
$\left.i{ }^{\circ}\right]$ & 80.50 & 80.51 & 1.0 (est.) \\
$\Omega_{1}$ & 8.77 & - & 0.10 (est.) \\
$\Omega_{2}$ & 4.00 & 3.98 & 0.02 (est.) \\
$F_{1}$ & 1.0 & - & - \\
$F_{2}$ & 1.0 & - & - \\
$T_{1}($ pole) $[\mathrm{K}]$ & 13000 & - & 1000 (est.) \\
$T_{2}($ pole) $[\mathrm{K}]$ & 5000 & 5989 & 200 (est.) \\
$\dot{M}\left[\mu M_{\odot} \mathrm{y}^{-1}\right]$ & 3.60 & 5.46 & 0.50 (est.) \\
$R_{A}\left[R_{\odot}\right]$ & 9.2 & 9.0 & 1.0 (est.) \\
$H_{V}\left[R_{\odot}\right]$ & 3.5 & 2.9 & 0.2 (est.) \\
\hline
\end{tabular}

the components) is below the accuracy of light curve modeling. Therefore, we can assume that these values have been constant over the past fifty years covered by the observations. Hence, it should be legitimate to fold all the observations into a single phase diagram using the quadratic ephemeris (Eq. (4)). We used the value of the period $P_{0}$, given in Table 3 , for the reference epoch as the input to BINSYN.

We combined the datasets (4), (7), (8), (9), and (11) from Table 1 into three groups corresponding to the Johnson's $U B V$ filters and used the steepest descent method together with BINSYN to obtain the best fit of the system parameters. The solution was found by fitting the parameters of the system simultaneously in all three passbands.

Table 4 lists initial and final values of the system parameters. For the albedo and gravity brightening coefficients, we used the standard values for a radiative envelope of the primary and a convective envelope of the secondary. The initial values of photospheric potentials $\left(\Omega_{1}\right.$ and $\left.\Omega_{2}\right)$ and inclination, were chosen by trial and error. The same is true for the outer radius and height of the disk $\left(R_{A}\right.$ and $\left.H_{V}\right)$. The inner radius of the disk, $R_{B}$, was set to be equal to that of the star.

Since we expect the secondary to be an evolved star that almost fills its Roche lobe, we assume synchronized rotation $\left(F_{2}=1\right)$. The situation is quite the opposite when we consider the primary. Because it might gain momentum from the accreting material, we expect it to rotate faster. However, initial trials showed that this parameter has no visible effect on the resulting light curve. This is beacuse the primary is almost invisible through the accretion disk surrounding it. To reflect this, we set the formal value of this parameter $F_{1}=1$, but its final value is completely uncertain. Our model does not offer the possibility to measure this parameter more accurately.

The calculated and observed light curves in three passbands are shown in Fig. 8. In Fig. 9, we show three views of UX Mon at different orbital phases. The shape of the large secondary corresponds to its Roche geometry close to the critical, Roche-lobe filling case. The primary star, which is mainly eclipsed by the disk, is outlined for enhanced clarity.

We also investigated the uncertainty in the parameters describing the geometry of the disk, $R_{A}$ and $H_{V}$. The quality of the model fit for the various values of both parameters in the neighborhood of the best-fit solution is shown in Fig. 10. One can see that the uncertainty in the height of the disk $H_{V}$ is much smaller 

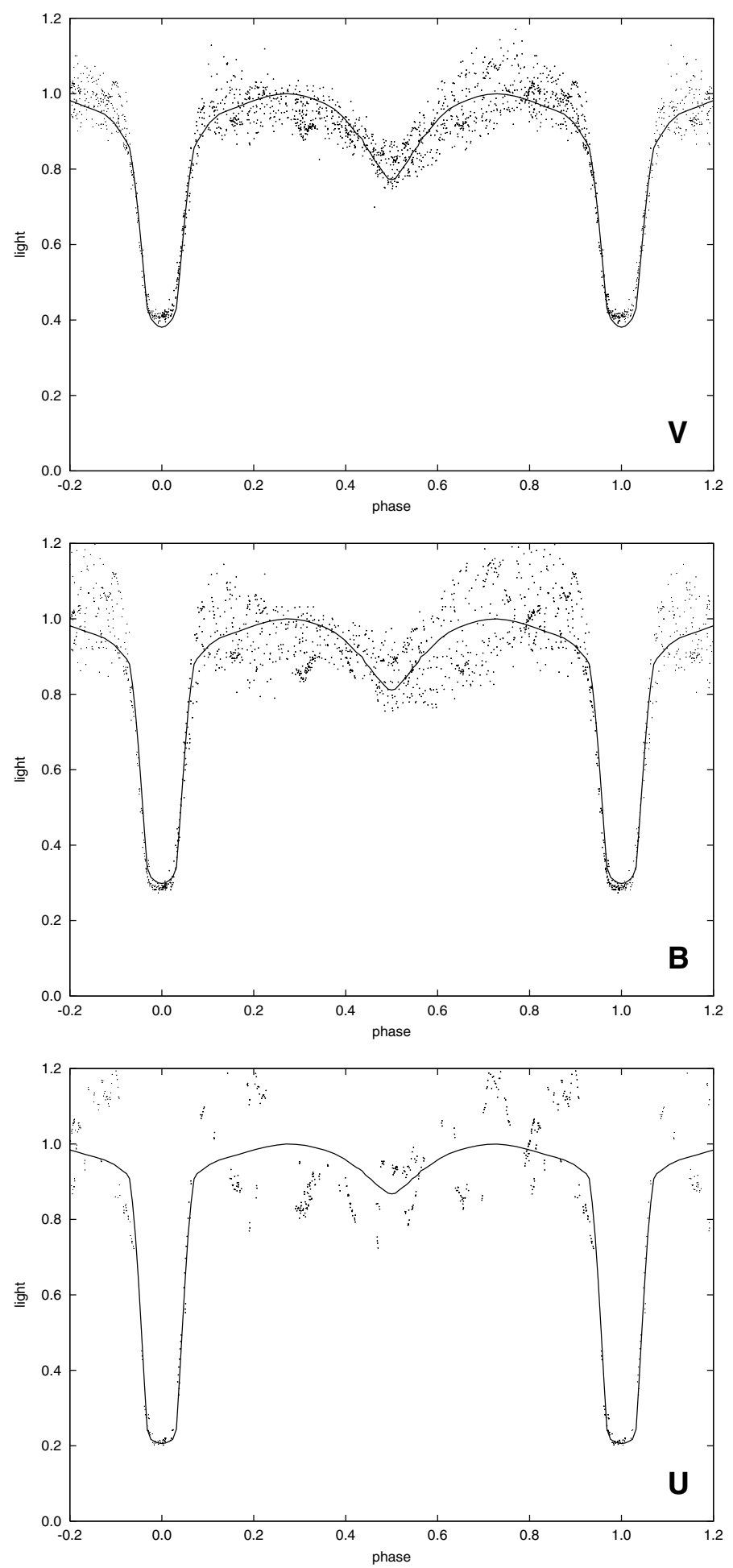

Fig. 8. Observed data and light curves calculated for the final set of parameters (Table 4)

than that of the outer disk radius $R_{A}$. Our estimates of the errors in both parameters, given in Table 4 , are based on the mapping presented in Fig. 10.

In Table 5, we give some important parameters of UX Mon derived from the values shown in Table 4. Note that, in both tables, certain errors are not indicated as estimated values, although they are calculated via a parameter whose error is estimated (mainly through connection with inclination, $i$, and mass ratio, $q$ ). Our estimate of the error in the inclination, $i$, is based
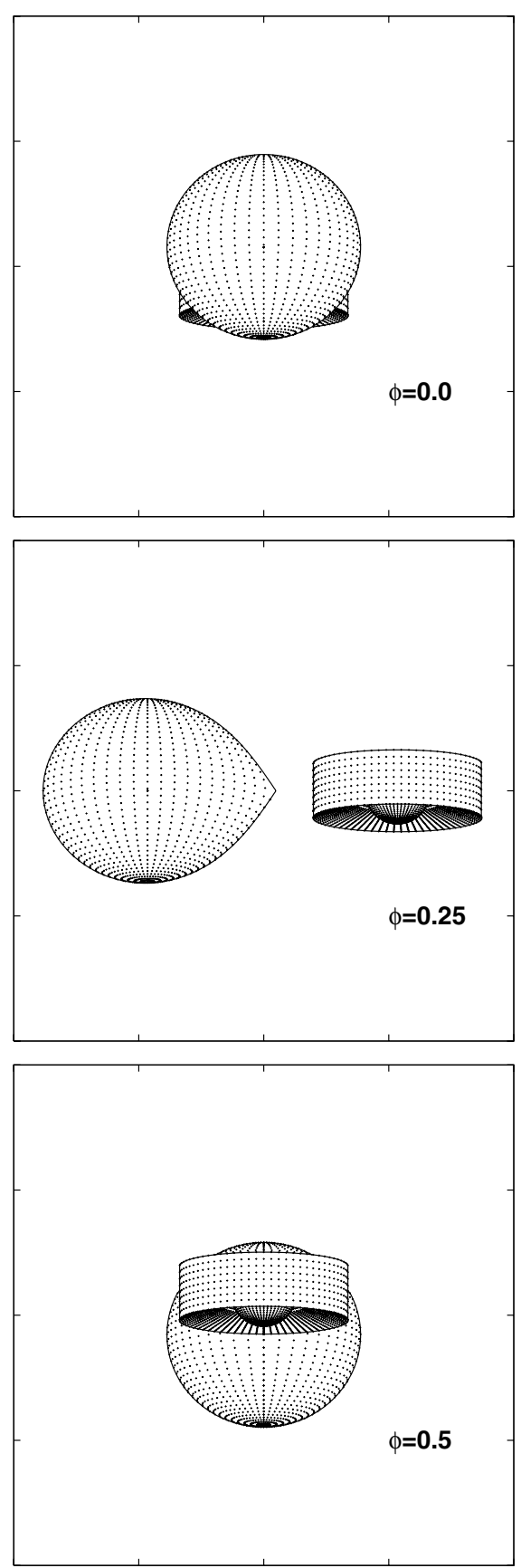

Fig. 9. Three views of the binary system UX Mon with an optically thick disk around the primary.

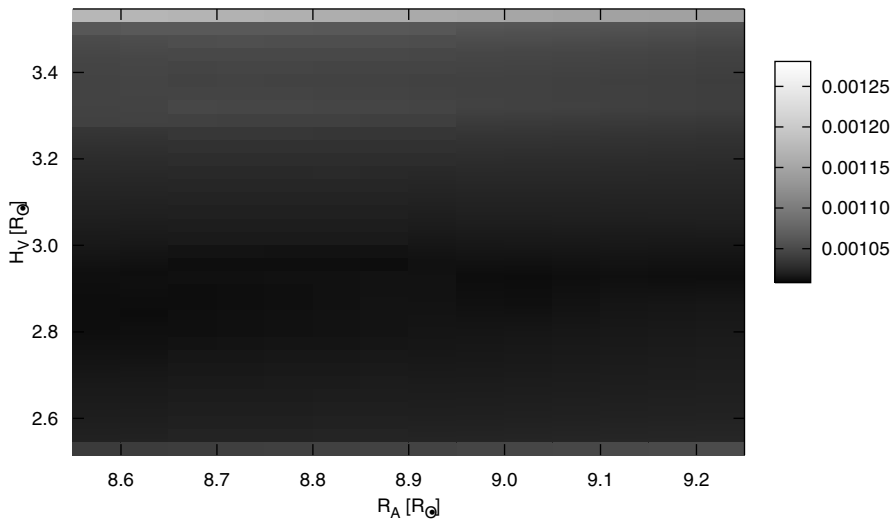

Fig. 10. A map plot of rms vs. $R_{A}$ and $H_{V}$ in the vicinity of the light curve solution. 
Table 5. Parameters of the UX Mon derived from the values listed in Table 4.

\begin{tabular}{lc}
\hline \hline Parameter & Value \\
\hline$M_{1}\left[M_{\odot}\right]$ & $3.38 \pm 0.40$ \\
$M_{2}\left[M_{\odot}\right]$ & $3.90 \pm 0.29$ \\
$R_{1}$ (pole) $\left[R_{\odot}\right]$ & $3.49 \pm 0.05$ \\
$R_{2}($ pole $)\left[R_{\odot}\right]$ & $9.80 \pm 0.03$ \\
$a\left[R_{\odot}\right]$ & $26.65 \pm 0.65$ \\
$\log g_{1}($ pole $)$ & $3.88 \pm 0.01$ \\
$\log g_{2}($ pole $)$ & $3.061 \pm 0.003$ \\
$T_{\mathrm{D}}($ rim $)[\mathrm{K}]$ & $6245 \pm 200$ \\
$T_{\mathrm{D}}($ face $)[\mathrm{K}]$ & $7487 \pm 200$ \\
$l_{1}$ & $0.39 \pm 0.03$ \\
$l_{2}$ & $0.44 \pm 0.01$ \\
$l_{\mathrm{D}}($ rim $)$ & $0.14 \pm 0.03$ \\
$l_{\mathrm{D}}($ face $)$ & $0.03 \pm 0.01$ \\
\hline
\end{tabular}

on the work of Linnell et al. (2006), who also used the BINSYN software package in their analysis. We admit, however, that UX Mon is a more complicated system because of the circumstellar matter around the primary. Consequently, the true uncertainty in the determination of $i$ could even be several degrees. The estimate of the error in the mass ratio, $q=0.05$, is subjective, based on our experience with the sensitivity of KOREL in finding the mass ratio (Sect 3.2). We also admit that the parameter mapping shown in Fig. 5 leaves us with some uncertainty regarding the error in $q$, which should not, however, be larger than \pm 0.1 . The values of $T_{\mathrm{D}}$ are the temperatures of the disk and $l_{1}, l_{2}, l_{\mathrm{D}}\left(\right.$ rim), and $l_{\mathrm{D}}$ (face) are luminosities relative to the system luminosity of the primary, secondary, rim of the disk, and face of the disk, respectively. Luminosities are calculated at phase $\phi=0.25$ for a wavelength of $\lambda=550 \mathrm{~nm}$.

\section{Discussion}

Using the stellar parameters obtained from our light curve solution, we can repeat the procedure of estimating the mass ratio, $q$, described at the beginning of Sect. 3.2. By first using, $T_{2}$ (back) = $5742 \mathrm{~K}$ and $\log g_{2}$ (back) $=2.83$, we get $\xi_{\mathrm{t}}=(0.5 \pm 0.25) \mathrm{km} \mathrm{s}^{-1}$ and $v_{2} \sin i=83.0 \pm 0.8 \mathrm{~km} \mathrm{~s}^{-1}$. The minimum $\chi^{2}$ obtained for this model is $\sim 1.5$, i.e. $\sim 1.25$ times smaller than the $\chi^{2}$ obtained from the model described at the beginning of Sect. 3.2.

In Fig. 11, we show the observed and synthetic spectra as well as the difference between the two. The match is not perfect, but the deviations can be attributed to the disk that is still visible.

We can again estimate the mass ratio, but this time using all values obtained in this paper: $K_{2}=104.6 \mathrm{~km} \mathrm{~s}^{-1}, i=80.5^{\circ}$, $P_{0}=5.9044365$ days, and $v_{2} \sin i=83.0 \mathrm{~km} \mathrm{~s}^{-1}$. This time the estimated mass ratio turns out to be $q_{\text {est }}=1.22$. This result is close to the value of $q=1.15 \pm 0.05$ that we found from the radial velocity analysis.

By looking at the differences between the measured and calculated RVs, we can use the Rossiter-McLaughlin effect to estimate the size of the secondary. We assume that the phase at which the discrepancies start $(\phi \approx 0.35)$ is the phase of the first contact of the secondary eclipse. The phase interval between the first contact and the center of the secondary eclipse is then $\Delta \phi \approx 0.15$. The distance covered by the star during this phase interval is $K_{2} P \Delta \phi$. On the other hand, we know from geometry of the system, that this distance is equal to $a_{2} \Delta \phi$, where $a_{2}$ is the distance of the secondary to the center of mass. We can

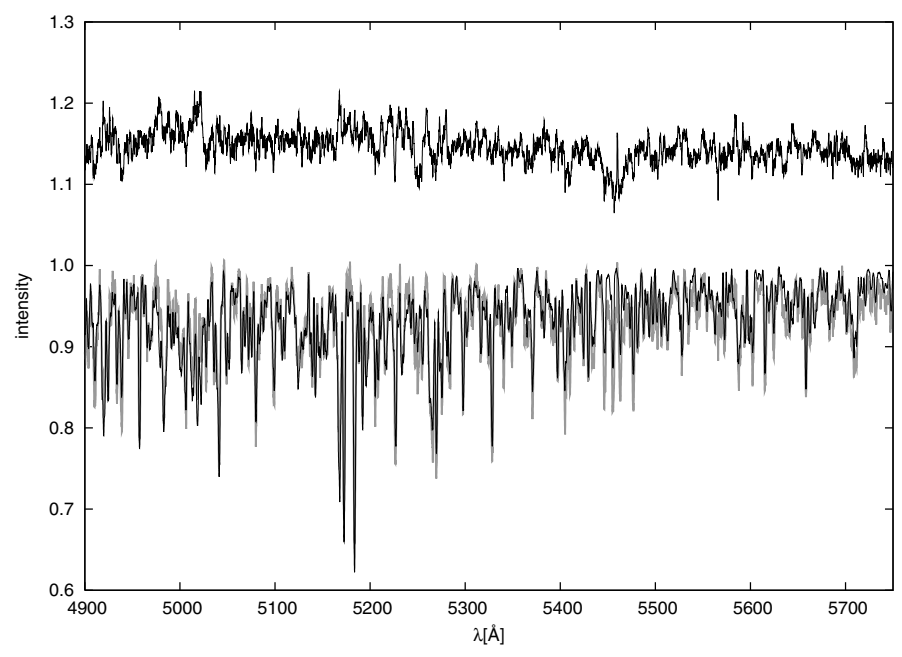

Fig. 11. Observed (gray) and calculated spectra (black, superimposed over the observed spectrum) for $T_{2}$ (back) $=5742 \mathrm{~K}$ and $\log g_{2}$ (back) $=$ 2.83. The difference spectrum is shifted upwards and shown in black.

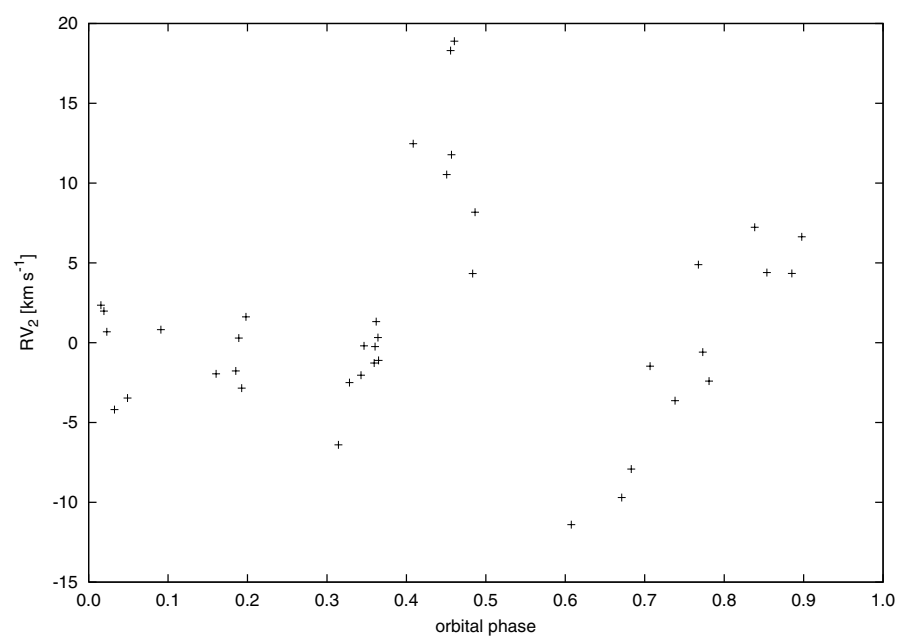

Fig. 12. Difference between the measured RVs and those calculated from Eq. (5).

eliminate $a_{2}$ by using $a_{2}=R_{2} / \sin (2 \pi \Delta \phi)$, assuming a circular orbit and ignoring the inclination. Finally we get

$R_{2}=\frac{K_{2} P}{2 \pi} \sin (2 \pi \Delta \phi) \approx 9.9 R_{\odot}$.

This value is close to the side radius of the disk that we found from our photometric solution $R_{2}$ (side) $=10.3 R_{\odot}$. From Fig. 13, we see that the eclipse starts at $\phi \approx 0.365$, which is a little later than we estimated from the RV measurements alone $(\phi \approx 0.35)$.

\section{Principal results and open questions}

The following principal results of our study lead to a substantial revision of the currently accepted model of the system:

1. The orbital period of the system is secularly decreasing at a rapid rate of $0.26 \mathrm{~s}$ per year.

2. The analysis of spectroscopic observations as well as the (plausible) assumption that the cooler, Roche-lobe filling component has its rotation synchronized with the orbital revolution both lead to the conclusion that the cooler component is the more massive of the two. 


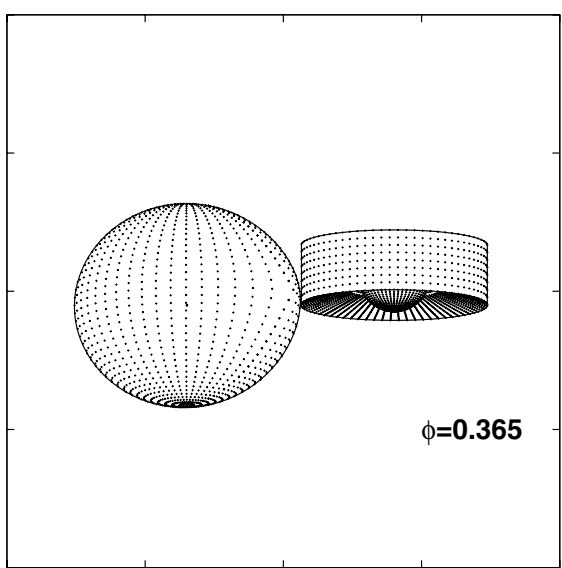

Fig. 13. Phase of first contact during secondary eclipse found from photometric solution.

3. Reasonable basic physical properties of the components were obtained via modeling the light curves on the premise that there is an optically thick accretion disk around the hotter component, which is eclipsed in the primary minimum. We admit, however, that the remaining uncertainties in some of the derived model parameters are still quite large.

4. The above findings identify UX Mon as a binary in a rare early stage of the mass exchange before the mass ratio reversal.

There are, however, still a few open questions and possible alternative views that need to be solved and clarified using new dedicated observations and more sophisticated modeling:

- There is no really satisfactory and self-consistent explanation of the true cause of non-orbital light changes clearly seen, for instance, in Fig. 8. These variations were very systematically investigated by Olson et al. (2009), who noted that the scatter in the light curve is largest in the phase interval $0.6-0.8$ from the primary minimum, i.e. around the elongation with component 1 receding from us (see their Fig. 2 or Fig. 8 here). These are the phases where the gas stream between the components can be seen projected on to the disk of the primary star. They therefore tentatively concluded that the light variations are related to variations in the rate of the mass transfer, possibly induced by the magnetic activity of the cool mass-losing star. There is, however, a very interesting plot in their Fig. 8 showing cyclic light variations at phases of totality during the primary minima with cycle lengths of some 800-1000 days. These variations are reminiscent of similar cyclic variations found for other strongly interacting binaries like RX Cas (517 d; Kalv 1979), $\beta$ Lyr (282 d; Harmanec et al. 1996) or AU Mon (417 d; Desmet et al. 2009). For all these three binaries, the light variations are probably strictly periodic and Desmet et al. (2009) argue that they are caused by variations in the circumbinary matter. All four systems, including UX Mon, are mass-exchanging binaries but only UX Mon is in the initial phase before the mass ratio reversal.

- There is also another aspect worth considering: Harmanec et al. (1996) showed that the bulk of the $\mathrm{H} \alpha$ emission in $\beta$ Lyr does not originate from the optically thick disk but from bipolar jets, oriented perpendicular to the orbital plane and originating from the region where the gas encircling the mass-gaining star hits the original gas stream flowing from the Roche-lobe filling component (Bisikalo et al. 2000).
Using only indirect arguments (mainly the phase offset of the RV curve of the $\mathrm{H} \alpha$ emission), Desmet et al. (2009) speculated about the possible presence of bipolar jets also for AU Mon. For the moment, this possibility cannot be excluded even for UX Mon.

- There are several possible ways to improve our understanding of UX Mon. First, spectrointerferometry of high resolution could show what the character and geometry of the medium responsible for the observed $\mathrm{H} \alpha$ emission is. Continuing systematic spectral and photometric variations could reveal possible long-term changes in the $\mathrm{H} \alpha$ emission and its relation to the - already known - long-term light changes. A hydrodynamical modeling based on the binary parameters obtained in this study could also show whether our tentative model is compatible with the predictions from the theory. Finally, a more sophisticated modeling of the light curves including the effects of various possible circumstellar structures, appears necessary, and not only for UX Mon.

- Considering how crucial UX Mon might be to achieving a clearer understanding of the process of large-scale mass exchange in this type of system, it seems obvious that the continuation of its systematic investigation is worth the effort.

Acknowledgements. We are very grateful to Prof. E. C. Olson and Mr. R. Meyer for providing us with their observations. We are also grateful to Dr. A. P. Linnell for providing us with the BINSYN software package. We thank Mr. D. Ondřich for making preliminary digitalization of the published photometric observations. We also thank Mrs. Laura Abrami and Mrs. Chiara Doz for supplying a copy of the Scaltriti paper, not available to us. We gratefully acknowledge the initial reduction of the Ondřejov CCD spectrograms by Dr. M. Šlechta. The first two Ondřejov spectra were obtained for us by Drs. M. Dovčiak and M. Wolf. We acknowledge the use of publicly available versions of the programs FOTEL and KOREL written by Dr. P. Hadrava. A constructive criticism of the original version of this study by the referee, Dr. Andrej Prša, helped to improve the presentation and is gratefully acknowledged. Dr. M. Wolf and Mr. D. Ondřich obtained a few observations of UX Mon at Hvar. The research of P.H and partly of H.B. was supported by the grants 205/06/0304 and P209/10/0715 of the Czech Science Foundation. P.H. was also supported from the Research Program MSM0021620860 Physical study of objects and processes in the solar system and in astrophysics of the Ministry of Education of the Czech Republic. We acknowledge the use of the electronic database from CDS Strasbourg and electronic bibliography maintained by the NASA/ADS system.

\section{References}

Andersen, J., Pavlovski, K., \& Piirola, V. 1989, A\&A, 215, 272

Bisikalo, D. V., Harmanec, P., Boyarchuk, A. A., Kuznetsov, O. A., \& Hadrava, P. 2000, A\&A, 353, 1009

Crawford, J. A. 1955, ApJ, 121, 71

Desmet, M., Frémat, Y., Baudin, F., et al. 2009, MNRAS, 1593

Gaposchkin, S. 1947, ApJ, 105, 258

Hadrava, P. 2004a, Publ. Astron. Inst. ASCR, 92, 1

Hadrava, P. 2004b, Publ. Astron. Inst. ASCR, 92, 15

Harmanec, P. 1988, Bull. Astron. Inst. Czechoslovakia, 39, 329

Harmanec, P. 1990, A\&A, 237, 91

Harmanec, P. 1998, A\&A, 335, 173

Harmanec, P., \& Scholz, G. 1993, A\&A, 279, 131

Harmanec, P., \& Božić, H. 2001, A\&A, 369, 1140

Harmanec, P., Horn, J., \& Juza, K. 1994, A\&AS, 104, 121

Harmanec, P., Morand, F., Bonneau, D., et al. 1996, A\&A, 312, 879

Hiltner, W. A., Struve, O., \& Jose, P. D. 1950, ApJ, 112, 504

Horn, J., Kubat, J., Harmanec, P., et al. 1996, A\&A, 309, 521

Kalv, P. 1979, Tartu Astrofuusika Observatoorium Teated, 58, 3

Linnell, A. P. 1984, ApJS, 54, 17

Linnell, A. P. 2000, MNRAS, 319, 255

Linnell, A. P., \& Hubeny, I. 1996, ApJ, 471, 958

Linnell, A. P., Harmanec, P., Koubský, P., et al. 2006, A\&A, 455, 1037

Lynds, C. R. 1956, PASP, 68, 339

Lynds, C. R. 1957a, ApJ, 126, 69 
Lynds, R. 1957b, AJ, 62, 24

Meyer, R. 2006, Open Eur. J. Variable Stars, 28, 1

Morton, D. C. 1960, ApJ, 132, 146

Olson, E. C., \& Etzel, P. B. 1995, AJ, 110, 2385

Olson, E. C., Henry, G. W., \& Etzel, P. B. 2009, AJ, 138, 1435

Ondřich, D., \& Harmanec, P. 2003, in WDS'03 Proc. Contributed Papers: Part III - Physics, ed. J. Safrankova, 502

Perryman, M. A. C., \& ESA, eds. 1997, The HIPPARCOS and TYCHO catalogues. Astrometric and photometric star catalogues derived from the ESA HIPPARCOS Space Astrometry Mission, ESA SP, 1200

Plavec, M. J. 1980, in Close Binary Stars: Observations and Interpretation, ed M. J. Plavec, D. M. Popper, \& R. K. Ulrich, IAU Symp., 88, 251

Plavec, M. J. 1992, in Nonisotropic and Variable Outflows from Stars, ed. L. Drissen, C. Leitherer, \& A. Nota, ASP Conf. Ser., 22, 47

Plavec, M., \& Koch, R. H. 1978, Inform. Bull. Variable Stars, 1482, 1

Pojmański, G. 1997, Acta Astron., 47, 467
Pojmański, G. 2001, in Small Telescope Astronomy on Global Scales, ed. B. Paczynski, W.-P. Chen, \& C. Lemme, ASP Conf. Ser., 246, IAU Colloq. 183,53

Scaltriti, F. 1973, Mem. Soc. Astron. Ital., 44, 387

Scaltriti, F. 1976, Inform. Bull. Variable Stars, 1154, 1

Shulyak, D., Tsymbal, V., Ryabchikova, T., Stütz, C., \& Weiss, W. W. 2004 A\&A, 428, 993

Škoda, P. 1996, in Astronomical Data Analysis Software and Systems V, ed. G. H. Jacoby, \& J. Barnes, ASP Conf. Ser., 101, 187

Struve, O. 1947, ApJ, 106, 255

Tsymbal, V. 1996, in M.A.S.S., Model Atmospheres and Spectrum Synthesis, ed. S. J. Adelman, F. Kupka, \& W. W. Weiss, ASP Conf. Ser., 108, 198

Umana, G., Catalano, S., \& Rodono, M. 1991, A\&A, 249, 217

Wood, F. B. 1957, AJ, 62, 327

Woods, I. E., \& Shapley, M. B. 1928, Harvard College Observatory Bulletin, 854,6 